\title{
Can We Place Central Venous Catheter Safely in Intensive Care Units?
}

\author{
Ashish Bhalla \\ Indian Journal of Critical Care Medicine (2020): 10.5005/jp-journals-10071-23510
}

Critical care medicine has blossomed into a vital specialty with tremendous advances in the equipment used, technological advances, and more importantly, the skill development in the critical care physicians. With advanced monitoring equipment based on solid evidence-based physics and physiology, the modern-day intensive care unit (ICU) looks nothing less than a space laboratory. A lot has changed in the way the interventions are being carried out in critically ill patients. As compared to a relatively hemodynamically stable patient in an operation theater, an intensivist is challenged by a critically ill, often crashing patient needing invasive monitoring in an already chaotic ICU environment. He/she is supposed to dig deep and put all his/her knowledge and skills to test, trying to save such patients.

Among many advances, noninvasive monitoring and the various multiparameter monitoring equipment have relatively made the monitoring easy in critical care units but in many cases vascular access is needed especially central venous catheterization (CVC). In critically ill patients, volume resuscitation, hyperalimentation, and infusion of vasoactive or inotropic drugs are important consideration and can be guided by noninvasive conventional parameters, such as pulse rate, blood pressure, and urine output measurement, or by monitoring central venous and arterial pressure. Vascular access intervention like CVC can be potentially associated with procedure-related and position-related complications. $^{1-3}$

There has been debate on where should CVC tip lie to provide adequate monitoring with minimum complication risk. ${ }^{4}$ In the good old days, several methods were used by physicians to guide correct depth of catheter insertion as no advanced screening methods were available. Peres' formula, which is calculated as height (in $\mathrm{cm}$ )/10 for the right internal jugular vein, is one such simple, widely used, and easy-to-remember formula. ${ }^{5}$ With the arrival of portable radiological imaging, use of portable chest X-rays and now picture archiving and communication system (PACS) have revolutionized the management of patients in critical care units. Radiological landmark formula uses anatomical landmarks like right sternoclavicular joint and the carina to calculate the length of catheter to be placed at superior vena cava and right atrium (SVCRA) junction. ${ }^{6}$ Simple calculation requires a chest $X$-ray to calculate the length of central venous catheter insertion in internal jugular vein. ${ }^{6}$ Use of anatomical landmarks for catheter insertion has solved the issue of misplacement of catheter tip to some extent but it has to be judiciously used. Blind dependence on these formulae can still cause misplacement of catheter tip leading to complication and fallacious readings. Therefore, an intensivist is always in the search of a better, reliable, safe formula to follow.

With advances in imaging technology, point of care ultrasonography (POCUS) has established itself as the imaging modality of choice in critical care units. ${ }^{7}$ Point of care ultrasonography
Department of Internal Medicine, Postgraduate Institute of Medical Education and Research, Chandigarh, India

Corresponding Author: Ashish Bhalla, Department of Internal Medicine, Postgraduate Institute of Medical Education and Research, Chandigarh, India, Phone: +91 9417023973, e-mail: bhalla.chd@gmail. com

How to cite this article: Bhalla A. Can We Place Central Venous Catheter Safely in Intensive Care Units? Indian J Crit Care Med 2020;24(7): 498-499.

Source of support: Nil

Conflict of interest: None

is both reliable, reproducible and gives the intensivist a window to peep inside a critically ill patient, to assess all vital organs, and to monitor physiological response to various interventions. ${ }^{7}$ In this era of evidence-based medicine, the papers like the one published by AR Manudeep, BP Manuja, and Dinesh Kumar, in this issue, are important articles as they reconfirm the importance of anatomical landmarks to guide the interventions. This paper also highlights the fact that just taking into consideration patient's height may not be appropriate but using hard anatomical landmarks like sternoclavicular joint and the carina in an individual is likely to be more specific guide to indicate SVC-RA junction fairly correctly during CVC placements. This can be an important tool in situations where the intensivist is forced to secure central venous access in the absence of POCUS or in the absence of having requisite skills to use POCUS.

One of the important messages from this paper is the fact that properly performed chest X-ray, which is widely available at most of the centers, can be easily utilized by an intensivist to guide catheter insertion accurately. This fact has been appropriately confirmed scientifically with transesophageal echocardiography used in this study to confirm the tip placement which is a very sensitive and specific imaging modality for cardiovascular imaging. This fact can further boost the confidence of the intensivist performing the central venous catheter placement. One has to remember that any intervention is operator dependent (knowledge, skill, and experience) and the formulae can only guide but cannot ensure safety and accuracy of catheter placement. Another important thing to remember while following radiological landmark formula is the fact that this is only applicable if we are trying to place CVC in right internal jugular and cannot be relied upon when using the left subclavian for CVC placement. ${ }^{8}$ With the widespread availability of POCUS or conventional sonography machines in most of the centers equipped to handle critical care patients, an image-guided procedure for central venous catheter placement and confirming the catheter tip should be aimed for, however, in desperate situations radiological formula can be a safe fallback option. 
Evidence-based confirmation of various formulae to help manage patients safely is the need of the hour and such studies are needed to build confidence in intensivist working in periphery with minimal radiological support for better and safer management of patients.

\section{References}

1. Chhabra L, Spodick DH. Complete heart block-an underappreciated serious complication of central venous catheter placement. J Electrocardiol 2012;45(6):790-792. DOI: 10.1016/ j.jelectrocard.2012.06.005

2. Robinson JF, Robinson WA, Cohn A, Garg K, Armstrong 2nd JD. Perforation of the great vessels during central venous line placement. Arch Intern Med 1995;155(11):1225-1228. DOI: 10.1001/ archinte.1995.00430110149016.

3. Collier PE, Blocker SH, Graff DM, Doyle P. Cardiac tamponade from central venous catheters. Am J Surg 1998;176(2):212-214. DOI: 10.1016/ S0002-9610(98)00171-8.
4. Fletcher SJ, Bodenham AR. Safe placement of central venous catheters: where should the tip of the catheter lie? $\mathrm{Br} J$ Anaesth 2000;85(2):188-191. DOI: 10.1093/bja/85.2.188.

5. Peres PW. Positioning central venous catheters-a prospective survey. Anaesth Intensive Care 1990;18(4):536-539. DOI: 10.1177/0310057X9001800422.

6. Ahn J, Kim I, Yang J, Lee I, Seo D, Kim S. Transoesophageal echocardiographic evaluation of central venous catheter positioning using Peres' formula or a radiological landmark-based approach: a prospective randomized single-centre study. $\mathrm{Br} J$ Anaesth 2017;118(2):215-222. DOI: 10.1093/bja/aew430.

7. Gillman LM, Kirkpatrick AW. Portable bedside ultrasound: the visual stethoscope of the 21st century Scandinavian. J Trauma, Resuscitat Emerg Med 2012;20(1):18. DOI: 10.1186/1757-7241-20-18.

8. Manudeep AR, Manjula BP, Dinesh Kumar US. Comparison of Peres' Formula and Radiological Landmark Formula for Optimal Depth of Insertion of Right Internal Jugular Venous Catheters. Indian J Crit Care Med 2020;24(7):527-530. 